Engineering Physics and Mathematics Division

Mathematical Sciences Section

\title{
THE FOURIER ANALYSIS TECHNIQUE AND EPSILON-PSEUDO-EIGENVALUES
}

\author{
June M. Donato
}

\begin{abstract}
Mathematical Sciences Section
Oak Ridge National Laboratory

P.O. Box 2008, Bldg. 6012

Oak Ridge, TN 37831-6367
\end{abstract}

Date Published: July 1993

Research was supported by the Applied Mathematical Sciences Research Program of the Office of Energy Research, U.S. Department of Energy.

Prepared by the Oak Ridge National Laboratory

Oak Ridge, Tennessee 37831 managed by

Martin Marietta Energy Systems, Inc.

for the

U.S. DEPARTMENT OF ENERGY

under Contract No. DE-AC-05-840R21400

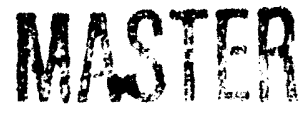




\title{
THE FOURIER ANALYSIS TECHNIQUE AND EPSILON-PSEUDC-EIGENVALUES
}

\author{
June M. Donato
}

\begin{abstract}
The spectral radii of iteration matrices and the spectra and condition numbers of preconditioned systems are important in forecasting the convergence rates for iterative methods. Unfortunately, the spectra of iteration matrices or preconditioned systems is rarely easily available. The Fourier analysis technique has been shown to be a useful tool in studying the effectiveness of iterative methods by determining approximate expressions for the eigenvalues or condition numbers of matrix systems.

For non-symmetric matrices the eigenvalues may be highly sensitive to perturbations. The spectral radii of nonsymmetri: iteration matrices may not give a numerically realistic indication of the convergence of the iterative method. Trefethen and others have presented a theory on the use of $\epsilon$-pseudo-eigenvalues in the study of matrix equations.

For Toeplitz matrices, we show that the theory of $\epsilon$-pseudo-eigenvalues includes the Fourier analysis technique as a limiting case. For non-Toeplitz matrices, the relationship is not clear. We shall examine this relationship for non-Toeplitz matrices that arise when studying preconditioned systems for methods applied to a two-dimensional discretized elliptic differential equation.
\end{abstract}




\section{Introduction}

The spectral radii of iteration matrices and the spectra and condition numbers of preconditioned systems are important in forecasting the convergence rates of iterative methods. A variety of methods are used in the analysis of such matrices $[1,12,13,19]$.

It is not always possible to determine analytic formulas describing the eigenvalues of a given matrix. Similarly, it is not always possible to determine reasonable analytic bounds on the extremal eigenvalues or the condition number of a matrix. In many cases where bounds have been determined, the analysis has been difficult or tedious $[2,9]$.

When exact eigenvalue analysis is used to obtain extremal eigenvalue bounds, we must be aware that for non-normal matrices that these eigenvalues can be highly sensitive to perturbations. Hence, the exact spectral radius or condition number of a non-normal iteration matrix may not give a realistic indication of the usefulness of the iterative method or preconditioner.

Motivated by the sensitivity of the eigenvalues to perturbations in the matrix, Trefethen and others have utilized a theory on $\epsilon$-pseudo-eigenvalues in the study of matrix equations. The $\epsilon$-pseudo-eigenvalues of non-normal matrices may portray radically different qualities than the exact eigenvalues for the matrix. See references [15] and [18].

Alternately, a heuristic technique based on Fourier analysis can be used to easily obtain eigenvalue approximations. The technique has been shown to be a useful heuristic when studying the effectiveness of iterative methods and preconditioners $[3,5,6,7,8,10]$. As yet no rigorous explanation of why this technique works so well for non-periodic non-constant diagonal matrices has been established. For symmetric Toeplitz matrices, the technique yields the true eigenvalue expressions, but it is does not always yield a good approximation for general matrices.

Herein, it is shown that for Toeplitz matrices the theory of $\epsilon$-pseudo-eigenvalues includes the Fourier analysis technique as a limiting case. Hence, the Fourier approximate eigenvalues serve as an approximation to the $\epsilon$-pseudo-eigenvalues.

For non-Toeplitz matrices, the relationship is not clear. Here, we consider nonToeplitz matrices that arise when studying preconditioned systems for methods applied to a two-dimensional discretized elliptic differential equation. We examine the relationship between the Fourier approximate eigenvalues and the $\epsilon$-pseudo-eigenvalues for these matrices.

The remainder of this paper is organized as follows. In Sections 2 and 3 we very briefly overview the Fourier analysis technique and concepts from the theory of $\epsilon$ pseudo-eigenvalues, respectively. For greater detail and a wide range of examples and applications see the references, especially $[6,15,18]$. In Section 4 we present the theorem linking the Fourier analysis technique and $\epsilon$-pseudo-eigenvalues for Toeplitz matrices. In Section 5 the spectra, Fourier approximate eigenvalues, and the $\epsilon$-pseudo-eigenvalues are graphically presented for a variety of Toeplitz matrices. In Section 6 we examine the relationship between the Fourier analysis technique and $\epsilon$-pseudo-eigenvalues by comparing results for preconditioned systems for a discretized two-dimensional elliptic partial differential equation. In Section 7 results and observations are summarized. 
Derivations of the Fourier approximate eigenvalues expressions are given in an appendix.

\section{Fourier Analysis}

Fourier analysis is a pervasive subject in all of mathematics. Here we are interested in how it can be used to determine eigenvalues or approximate eigenvalues of a given matrix. Consider a one-dimensional constant coefficient elliptic partial differential equation (PDE) with periodic boundary conditions discretized on a uniform grid with $N$ internal grid points. Let $A u=b$ denote the resulting matrix system where $A$ is an $(N+1) \times(N+1)$ matrix.

Let $u^{(s)}$ be a column vector of length $N+1$ composed of the one-dimensional Fourier exponential modes. ${ }^{1}$ The $j^{\text {th }}$ component of $u^{(s)}$ is given by

$$
u_{j}^{(s)}=e^{i j \theta_{s}} \text { where } \theta_{s}=\frac{2 \pi s}{N+1}, \quad j=0,1, \ldots, N, \quad s=0,1, \ldots, N .
$$

The $N+1$ vectors $\left\{u^{(s)}: s=0,1, \ldots, N\right\}$, called the Fourier vectors, are eigenvectors of such a circulant matrix $A$. The fact that we know a basis for such a matrix makes it quite easy to determine an analytic formula for its eigenvalues.

Although elliptic PDEs rarely yield circulant matrices when discretized, Fourier analysis is often used $[5,6,7,8]$ in the same way that von Neumann analysis is used for time-dependent systems [16], and local mode analysis is used for multigrid methods [4].

The steps of the Fourier analysis technique as given by [6] for an elliptic partial differential equation are summarized as follows:

(a) Treat the matrices involved as if they came from periodic problems. This may involve ignoring the original boundary conditions of the problem and/or extending the original matrix.

(b) Force the matrices to have constant diagonal entries. This may entail using an asymptotic value for the diagonal entries, as in the case for the ILU preconditioner.

(c) From concepts developed in [6] use the relation $h_{p}=2 h_{d}$ to relate the periodic mesh size to the Dirichlet mesh size.

After performing the above steps, we would have a circulant matrix whose eigen vectors are the Fourier vectors of the appropriate dimension. We are then able to use exact Fourier analysis on the altered matrix to determine approximations of its minimum or maximum eigenvalues or to observe the general shape of its spectrum. This is done simply by computing

$$
\tilde{A} u^{(s)}=\tilde{\lambda}_{s} u^{(s)}
$$

\footnotetext{
${ }^{1}$ Similarly, for a constant coefficient matrix with Dirichlet or Neumann boundary conditions we would use the Fourier sine or cosine modes, respectively, as eigenvactor components.
} 
where $\tilde{A}$ represents the modified matrix and $\bar{\lambda}_{s}$ denotes the $s^{\text {th }}$ eigenvalue of $\tilde{A}$. Since $\tilde{A}$ is constant diagonal, this computation can be done easily using component or stencil form. The eigenvalue, $\bar{\lambda}_{s}$, is a function of $\theta_{s}^{(p)}=2 \pi s h_{p}=2 \pi s /\left(n_{p}+1\right)$ where $n_{p}=$ $2 n_{d}+1$ and $s \in\left\{1, \ldots, n_{p}\right\}$. Thus, $\theta_{s}^{(p)} \in(0,2 \pi)$. $\tilde{A}:$

The Fourier approximate eigenvalues of $A, \hat{\Lambda}$, are then given by the eigenvalues of

$$
\hat{\lambda}_{s}=\hat{\lambda}_{s}(A)=\tilde{\lambda}_{s} .
$$

The degenerate eigenvalue, $\bar{\lambda}_{s}=0$, is ignored when doing analysis for a problem with Dirichlet boundary conditions.

For higher dimensional problems, the same steps as given above would be followed to generate the approximating matrix $\tilde{A}$. However, the appropriate dimensioned Fourier modes would form the components of the eigenvectors of $\tilde{A}$. For example, suppose we have a two-dimensional problem discretized on a uniform grid with $N$ internal grid points in each direction. Then the Fourier $(j, k)^{t h}$ component of the $(s, t)^{t h}$ eigenvector would be given by

$$
u_{j, k}^{(s, t)}=e^{i j \theta_{s}} e^{i k \phi_{t}}, \quad 0 \leq s, t \leq n,
$$

where $\theta_{s}=2 \pi s h, \phi_{t}=2 \pi t h, h=1 /(n+1)$. As above, we would then determine $\tilde{\lambda}_{s, t}$ from

$$
\tilde{A} u^{(s, t)}=\tilde{\lambda}_{s, t} u^{(s, t)} \text {. }
$$

As in the one-dimensional case, the singular cases with $s=0$ or $t=0$ are: :gnored in the analysis of problems with Dirichlet boundary conditions.

\section{3. $\epsilon$-pseudo-eigenvalues}

For non-hermitian matrices, the eigenvalues of the matrix may be highly sensitive to perturbations. Hence, when analyzing a matrix to determine its liehavior as an iteration matrix or as a preconditioner, the true eigenvalues of the matrix may be misleading. In fact, we are more interested in the behavior of the eigenvalues when the matrix $A$ is perturbed.

This is the connection to the theory of $\epsilon$-pseudo-eigenvalues as presented in references $[15,17,18]$. There are several theoretically equivalent definitions for $\epsilon$-pseudoeigenvalues. We will use the following definition from [18].

Definition: Given $\epsilon>0, \lambda \in \mathrm{C}$ is an $\epsilon$-pseudo-eigenvalue of the $N \times N$ matrix $A$ if $\lambda$ is an eigenvalue of $A+E$ for some $E \in \mathrm{C}^{N \times N}$ with $\|E\| \leq \epsilon$. The set of all $\epsilon$-pseudo-eigenvalues of $A$, called the $\epsilon$-pseudo-spectrum, is denoted $\Lambda_{\epsilon}(A)$ or simply $\Lambda_{\epsilon}$.

Rather than examine the exact eigenvalues of a non-normal matrix $A$ we want to examine $\Lambda_{\epsilon}$. However, computing $\Lambda_{\epsilon}(A)$ using the definition is not always desirable or feasible for large $N$. But, Reichel and Trefethen [15] have observed a relationship between the $\epsilon$-pseudo-spectrum and the union of three sets for Toeplitz matrices. The following is an overview of one of their results. 
Consider the matrix problem $A u=b$ where $A$ is a Toeplitz matrix

$$
A=\left(\begin{array}{cccc}
a_{0} & a_{1} & \cdots & a_{N} \\
a_{-1} & a_{0} & \ddots & \vdots \\
\vdots & \ddots & \ddots & a_{1} \\
a_{-N} & \cdots & a_{-1} & a_{0}
\end{array}\right)
$$

A matrix of this form could have arisen from a finite-difference or finite-element discretization of a one-dimensional self-adjoint PDE. The symbol [15] of this matrix is given by $f(z)=\sum_{k=-N}^{N} a_{k} z^{k}$. The fundamental observation of [1.5] is that for large $N$ and small $\epsilon, \Lambda_{c}$ looks approximately like the union of three sets:

$$
\Lambda_{\epsilon} \simeq \Omega_{\mathrm{r}} \cup \Omega^{R} \cup\left(\Lambda+\Delta_{\epsilon}\right),
$$

where

$$
\begin{aligned}
\Omega_{r} & =\left\{z \in \mathrm{C}: I\left(f\left(S_{r}\right), z\right)>0\right\} \\
\Omega^{R} & =\left\{z \in \mathrm{C}: I\left(f\left(S_{R}\right), z\right)<0\right\} \\
S_{r} & =\text { circle of radius } r, r=(\epsilon / c)^{1 / N} \\
S_{R} & =\text { circle of radius } R, R=(\epsilon / C)^{-1 / N} \\
I(f, z) & =\text { winding number of } f \text { about } z \\
\Lambda & =\text { the eigenvalues of the matrix } A \\
\Lambda+\Delta_{\epsilon} & =\text { union of } \epsilon \text {-balls about the eigenvalues of the matrix } A
\end{aligned}
$$

The values $c$ and $C[15]$ are generally taken to be 1 .

The images of $S_{r}$ and $S_{R}, f\left(S_{r}\right)$ and $f\left(S_{R}\right)$, are easily computed. Typically $\Omega_{r}$ and $\Omega^{R}$ provide a good envelope for $\Lambda_{\varepsilon}(A)$. Hence, by computing the regions enclosed by $f\left(S_{r}\right)$ and $f\left(S_{R}\right)$, we can get a general idea of the behavior of the matrix without the computationally expensive task of computing $\Lambda$ or $\Lambda_{c}(A)$. This is certainly advantageous in the analysis of iterative methods and preconditioners.

\section{The link between the Fourier technique and $\epsilon$-pseudo-eigenvalues}

In this section it is shown that the Fourier analysis technique yields a limiting expression for the boundaries of the regions $\Omega_{\tau}$ and $\Omega^{R}$.

TH EOREM: For the general one-dimensional Toeplitz matrix the boundary defined by the Fourier approximate eigenvalue expression, $\hat{\lambda}(A)$, is a limiting case of the boundaries of $\Omega_{r}$ and $\Omega^{R}$ as $r, R \rightarrow 1$. 
Proof. Consider again the Toeplitz matrix (1). We have already noted that the symbol of this matrix is given by

$$
f(z)=\sum_{k=-N}^{N} a_{k} z^{k}
$$

From (2) we are interested in the boundaries of the regions $\Omega_{r}$ and $\Omega^{R}$, which are determined by the images of $S_{r}$ and $S_{R}$ via the symbol $f(z)$. The image of $S_{r}$ is given by

$$
f\left(S_{r}\right)=\left\{z=f\left(r e^{i \theta}\right): \theta \in[0,2 \pi]\right\},
$$

where

$$
f\left(r e^{i \theta}\right)=\sum_{k=-N}^{N} a_{k}\left(r e^{i \theta}\right)^{k}=\sum_{k=-N}^{N} r^{k} a_{k}\left(e^{i \theta}\right)^{k}
$$

with $r=\epsilon^{1 / N}$, and similarly for $f\left(S_{R}\right)$ using $R=\epsilon^{-1 / N}$ instead of $r$. As $N \rightarrow \infty$, we have $r \rightarrow 1, R \rightarrow 1$ since $\epsilon \ll 1$.

To apply the Fourier analysis technique to this Toeplitz matrix (1) we follow the steps outlined earlier. The circulant version of the matrix $A$ is

$$
\tilde{A}=\left(\begin{array}{lllllll}
a_{0} & \cdots & a_{N-1} & a_{N} & a_{-N} & \cdots & a_{-1} \\
\vdots & \ddots & \vdots & \vdots & \vdots & \ddots & \vdots \\
a_{N} & \cdots & a_{0} & a_{1} & a_{2} & \cdots & a_{-N} \\
a_{-N} & \cdots & a_{-1} & a_{0} & a_{1} & \cdots & a_{N} \\
\vdots & \ddots & \vdots & \vdots & \vdots & & \vdots \\
a_{-1} & \cdots & a_{-N} & a_{N} & a_{N-1} & \cdots & a_{0}
\end{array}\right)
$$

where $\tilde{A}$ is an order $2 N+1$ matrix.

We calculate the $j^{\text {th }}$ component of

$$
\tilde{A} u^{(s)}=\tilde{\lambda}_{s} u^{(s)}
$$

to get

$$
\begin{aligned}
\tilde{A} u_{j}^{(s)} & =a_{0} e^{i j \theta_{s}^{(p)}}+a_{1} e^{i(j+1) \theta_{s}^{(p)}}+\cdots+a_{N} e^{i(j+N) \theta_{s}^{(p)}} \\
& +a_{-1} e^{i(j-1) \theta_{s}^{(p)}}+\cdots+a_{-N} e^{i(j-N) \theta_{s}^{(p)}} \\
= & \left(\sum_{k=-N}^{N} a_{k} e^{i k \theta_{s}^{(p)}}\right) u_{j}^{(s)} \\
= & \tilde{\lambda}_{s} u_{j}^{(s)}
\end{aligned}
$$


The Fourier approximate eigenvalues of $A$ are then given by the eigenvalues of $\tilde{A}$,

$$
\hat{\lambda}_{s}(A)=\bar{\lambda}_{s}=\sum_{k=-N}^{N} a_{k}\left(e^{i \theta_{s}^{(p)}}\right)^{k},
$$

where $\theta_{s}^{(p)} \in(0,2 \pi)$.

By comparing the Fourier approximate eigenvalues of $A$ in (5) to the images of $S_{r}$ and $S_{R}$ via the symbol for $A$ in (4), we see that (5) is a discrete version of (4) where $r=1$. And as already noted, $r=1$ and $R=1$ are the limiting values as $N \rightarrow \infty$.

Thus the theory of $\epsilon$-pseudo-eigenvalues includes as a limiting case the Fourier analysis technique. This result is certainly related to the fact that exact Fourier analysis leads to samples of the curve $f(|z|=1)$ for Toeplitz systems. In Reichel and Trefethen [15], it is stated that

For small $\epsilon$ and large $N$, the $\epsilon$-pseudospectrum $\Lambda_{\epsilon}$ of a Toeplitz matrix is roughly the same as the spectrum of the associated Toeplitz operator, namely, a region in the complex plane bounded by the curve $f(S)$, where $f(z)$ is the symbol of the matrix.

The important and interesting concept here is that the theory of $\epsilon$-pseudo-eigenvalues may provide the explanation as to why the the approximate Fourier analysis technique has yielded good approximations even for situations where Fourier analysis does not strictly apply.

For the non-limiting case, the boundary formed by the Fourier approximate eigenvalues lies between $\Omega_{r}$ and $\Omega^{R}$. And so the Fourier boundary would enclose most (if not all) of the $\epsilon$-pseudo-eigenvalues. Empirically, we will see that it seems to include all of the $\epsilon$-pseudo-eigenvalues.

\section{Toeplitz Examples}

In this section, some Toeplitz examples are given that demonstrate the relationship between $\epsilon$-pseudo-eigenvalues regions and the boundary defined via the Fourier approximate eigenvalues. In these examples, we use $N=100$ for the order of the matrix $A$ and $\epsilon=10^{-4}$. First, we consider two Toeplitz matrices studied in [15]. After these examples, we consider Toeplitz matrices arising from the discretization of a one-dimensional second-order differential equation.

In each of these pictures, the true eigenvalues, the $\epsilon$-pseudo-eigenvalues using a set of five randomiy generated perturbation matrices, $f\left(S_{r}\right), f\left(S_{R}\right)$, and the Fourier approximate eigenvalues are plotted. See the legend given in Table 1.

In each of these pictures we see that the $\epsilon$-pseudo-eigenvalues are enclosed by $\Omega^{R}$, which is surrounded by the Fourier boundary.

In Figure 1, example (3.8) of [15] is plotted along with the Fourier approximate 
eigenvalues. The matrix is

$$
A=\left(\begin{array}{cccc}
0 & 2 & & \\
1 & \ddots & \ddots & \\
& \ddots & \ddots & 2 \\
& & 1 & 0
\end{array}\right) .
$$

In Figure 1, $f\left(S_{r}\right)$ provides the tighter bound on the $\epsilon$-pseudo-eigenvalues. However, we still have the Fourier boundary between $f\left(S_{r}\right)$ and $f\left(S_{R}\right)$.

Figure 2 shows the regions for the Bull's head example [15] for the matrix

$$
A=\left(\begin{array}{cccccccc}
0 & 0 & 1 & .7 & & & & \\
2 i & 0 & 0 & 1 & .7 & & & \\
0 & 2 i & 0 & 0 & 1 & .7 & & \\
& \ddots & \ddots & \ddots & \ddots & \ddots & \ddots & \\
& & 0 & 2 i & 0 & 0 & 1 & .7 \\
& & & 0 & 2 i & 0 & 0 & 1 \\
& & & & 0 & 2 i & 0 & 0 \\
& & & & & 0 & 2 i & 0
\end{array}\right) .
$$

The regions depicted in Figure 2 are more complex, but it is still easy to see that the Fourier boundary lies "in-between" $\Omega_{r}$ and $\Omega^{R}$ and that the Fourier boundary encloses the $\epsilon$-pseudo-eigenvalues.

\begin{tabular}{ll} 
Symbol & Item Represented \\
\hline solid line & $\Omega_{r}$ \\
dashed line & $\Omega^{R}$ \\
0 & $\epsilon$-pseudo-eigenvalues \\
$\times$ & Fourier approximate eigenvalues \\
$*$ & eigenvalues \\
\hline
\end{tabular}

Table 1: Legend for $\hat{\Lambda}$ and $\Lambda_{\epsilon}$ figures 


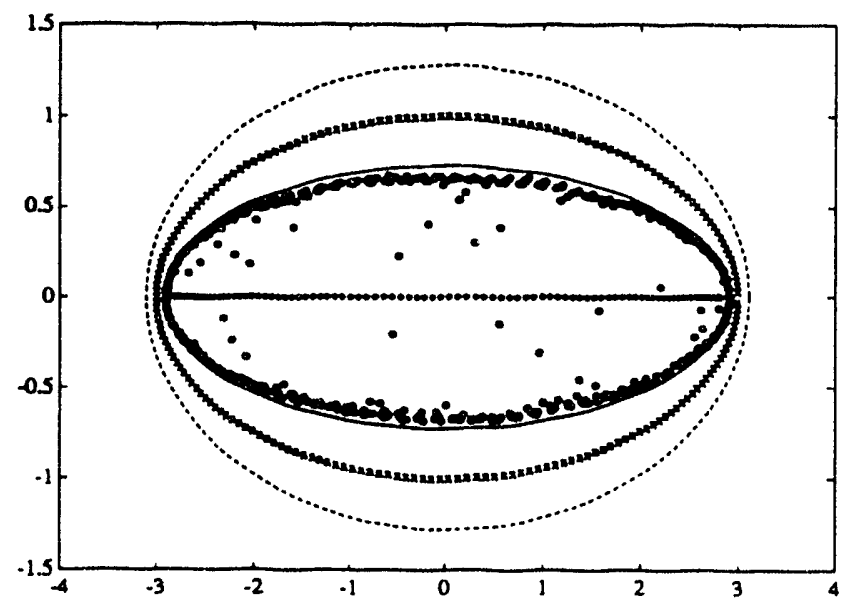

Figure 1: Regions for matrix $A$ of (6)

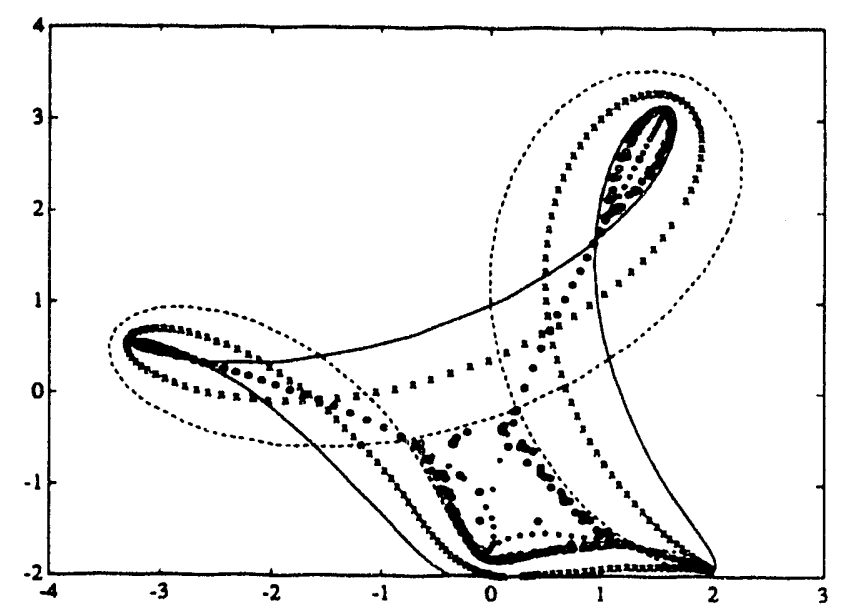

Figure 2: Bull's Head example 
Next we consider the Fourier approximate eigenvalues and the $\epsilon$-pseudo-spectra for the following one-dimensional second-order differential equation

$$
\begin{aligned}
& -u_{x x}+\gamma u_{x}=f, \quad \gamma>0, \\
& u(0)=u(1)=0,
\end{aligned}
$$

on $\Omega=[0,1]$. The region $\Omega$ is divided into $n+1$ uniform intervals of mesh size $h=\frac{1}{n+1}$, and centered differences for $u_{x x}$ and upwind differencing for $\gamma u_{x}$ are used. We get the matrix equation

$$
A u=b, \quad A \in \mathbf{R}^{n \times n}
$$

where $A$ is an $N \times N, N=n^{2}$, tridiagonal matrix of the form

$$
A=\left(\begin{array}{cccc}
a & b & & \\
c & \ddots & \ddots & \\
& \ddots & \ddots & b \\
& & c & a
\end{array}\right)
$$

with $a=2+\gamma h, b=-1$, and $c=-1-\gamma h$. In stencil form it is given by

$$
[-1-\gamma h, 2+\gamma h,-1] \text {. }
$$

The Fourier approximate eigenvalues of $A$ are

$$
\hat{\lambda}_{s}(A)=a+b e^{i \theta_{s}}+c e^{-i \theta_{s}}, \quad \theta_{s} \in(0,2 \pi),
$$

and the symbol of the matrix is

$$
f(z)=a+b z+c z^{-1} .
$$

In Figures 3-6, we use this ronsymmetric problem (7) to demonstrate the relation between the true eigenvalues of the problem and the Fourier and $\epsilon$-pseudo-eigenvalues. The nonsymmetry of the problem is varied by altering the value of the parameter $\gamma$. Again, $N=100$ and $\epsilon=10^{-4}$. 


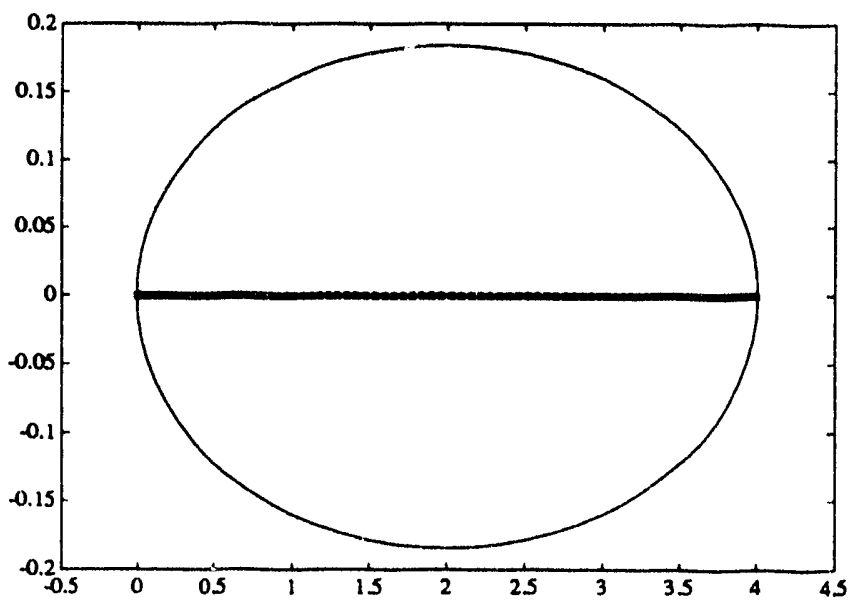

Figure 3: Regions for (7) with $\gamma=0$.

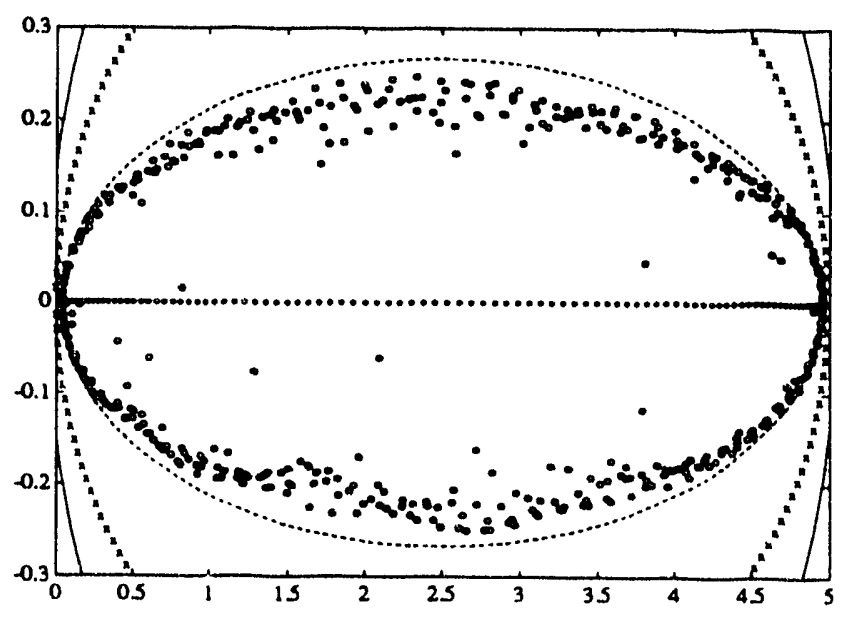

Figure 4: Regions for (7) with $\gamma=50$. 


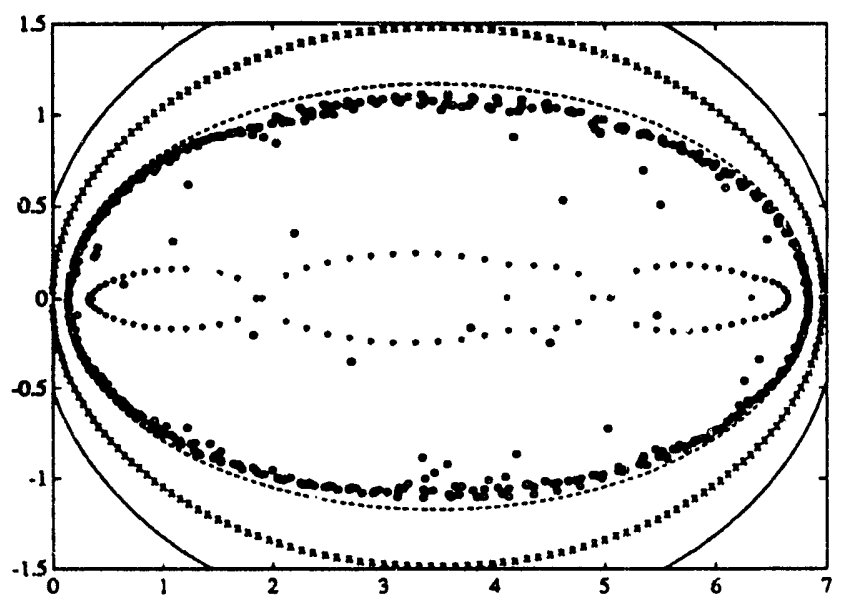

Figure 5: Regions for (7) with $\gamma=150$.

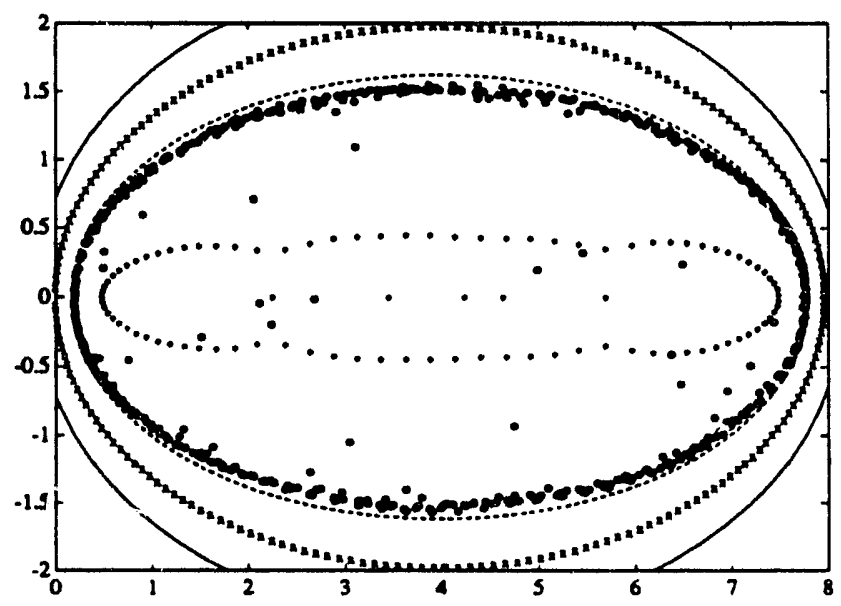

Figure 6: Regions for (7) with $\gamma=200$. 


\section{Non-Toeplitz Examples}

In the previous section, a relationship was shown to exist between the Fourier approximite eigenvalues and the $\epsilon$-pseudo-eigenvalues for Toeplitz matrices. Untortunately, the proof relating the Fourier approximate eigenvalues and the $\epsilon$-pseudo-eigenvalues bounding regions can not be immediately generalized to non-Toeplitz systems.

In this section, we examine pictorially the relationship between the $\epsilon$-pseudo-eigenvalues and Fourier approximate eigenvalues of certain non-Toeplitz matrices. These matrices will arise from the preconditioning of a discretized two-dimensional elliptic partial differential equation. We use the Jacobi and SOR $(w)$ splitting matrices and the ILU preconditioner.

The model two-dimensional parameterized elliptic partial differential equation that we consider is

$$
\begin{array}{rlll}
-\Delta u+\alpha u_{x}+\beta u_{y} & =f & \text { on } & \Omega=[0,1] \times[0,1] \\
u & =0 & \text { on } & \partial \Omega
\end{array}
$$

where $\Omega$ is partitioned into an uniform grid with $n$ interior grid points in each direction having a mesh size of $h=\frac{1}{n+1}$. Centered differences are used for the Laplacian and upwind differencing is used for the convection terms. The equation for the $(j, k)^{\text {th }}$ grid value of $u$ is given by

$$
a u_{j, k}+b u_{j+1, k}+c u_{j, k+1}+d u_{j-1, k}+\ell u_{j, k-1}=h^{2} f_{j, k},
$$

where $a=4+(\alpha+\beta) h, b=c=-1, d=-1-\alpha h, \ell=-1-\beta h$. Using the rowwise natural ordering for the components of $u$, the resulting scaled discretized system is given by

$$
A u=h^{2} f,
$$

where $A$ is an $N \times N$ matrix, $N=\pi^{2}$, in stencil form given by

$$
A=\left[\begin{array}{ccc}
\cdot & c & \cdot \\
d & a & b \\
\cdot & \ell & \cdot
\end{array}\right]=\left[\begin{array}{ccc}
\cdot & -1 & \cdot \\
-1-\alpha h & 4+(\alpha+\beta) h & -1 \\
\cdot & -1-\beta h & \cdot
\end{array}\right]
$$

While this matrix is close to being Toeplitz, it is not because the Dirichlet boundary conditions introduce zeros entries in the super and sub-diagonals. Nor will the resulting preconditioned systems be Toeplitz. So, we can no longer directly use the ReichelTrefethen observation for Toeplitz matrices, and the regions $\Omega_{r}$ and $\Omega^{R}$ will not be plotted. We compute the 6 -pseudo-eigenvalues via the definition given in Section 3 .

In Figure 7, there are three groups of three pictures corresponding to the three methods applied to the discretized system with parameter values of $\alpha=\beta=0$. In each set of three, we plot (from left to right) the the computed eigenvalues, the $\epsilon$-pseudoeigenvalues, and the Fourier approximate eigenvalues for the matrix $M^{-1} A$.

The first set of three corresponds to $M$ being the diagonal of $A$ (the Jacobi splitting matrix or preconditioner), the second set is for $M$ corresponding to the splitting matrix for $\operatorname{SOR}(w)$, and the third set for $M$ being the ILU preconditioner. 
This scheme is repeated for parameter values of $\alpha=5, \beta=1$ in Figure 8 and for parameter values of $\alpha=\beta=5$ in Figure 9 .

In computing the Fourier approximate eigenvalues for $A$ and its preconditioned systems, the two-dimensional Fourier exponential modes are used in the Fourier analysis technique.

The $\epsilon$-pseudo-eigenvalues are computed according to the definition given in section 3 by calculating the eigenvalues of $A+E$ for five randomly generated perturbation matrices with $\|E\| \leq \epsilon$. To compare the plots for the Fourier approximate eigenvalues and the $\epsilon$-pseudo-eigen values we must choose a reasonable value of $\epsilon$. For a polynomial $p_{n}(z)$ we have the relationship [14],

$$
\left\|p_{n}(z)\right\|_{\Lambda} \leq\left\|p_{n}(A)\right\| \leq \frac{L}{2 \pi \epsilon}\left\|p_{n}(z)\right\|_{\Lambda_{\epsilon}}
$$

where $L$ is the arc length of the boundary of $\epsilon$-pseudo-eigenvalues. Rather than compute the arc length, which is a ncin-trivial process, we choose that value of $\epsilon$ that reasonably scales the $\epsilon$-pseudo-eigenvalite plot. These values are given in Table 2.

\begin{tabular}{|l|c|c|c|}
\hline & $\alpha=\beta=0$ & $\alpha=5, \beta=1$ & $\alpha=\beta=5$ \\
\hline Jacobi & 0.10 & 0.10 & 0.10 \\
\hline SOR & 0.10 & 0.10 & 0.10 \\
\hline ILU & 0.01 & 0.001 & 0.01 \\
\hline
\end{tabular}

Table 2: $\epsilon$ values used in the $\epsilon$-pseudo-eigenvalue plots

It is obvious from these pictures that the Fourier approximate eigenvalues and the $\epsilon$-pseudo-eigenvalues can vary quite drastically in appearance when compared to the actual eigenvalues. Yet, the Fourier approximate eigenvalues mimic some of the clustering behavior of the $\epsilon$-pseudo-eigenvalues and does well in most instances to approximate the shape and extremal values of the $\epsilon$-pseudo-eigenvalues.

If it is indeed more critical to examine $\epsilon$-pseudo-eigenvalues when analyzing and designing iterative methods and preconditioners, then the Fourier analysis technique has several advantages. The Fourier approximate eigenvalues capture much of the clustering and bounding information of the $\epsilon$-pseudo-eigenvalues, but with significantly less computational effort. To compute the $\epsilon$-pseudo-eigenvalues we actually must compute the eigenvalues of $A+E$ for several randomly generated perturbal, in matrices. For each perturbation matrix this is an $O\left(N^{3}\right)$ operation for an $N \times N$ matrix. Computing the Fourier approximate eigenvalues is $O\left(N^{2}\right)$. In addition, the Fourier approximate eigenvalues are computed from the Fourier expression, which can be analyzed independently. This allows a researcher to tune a preconditioner to have a desired behavior. 

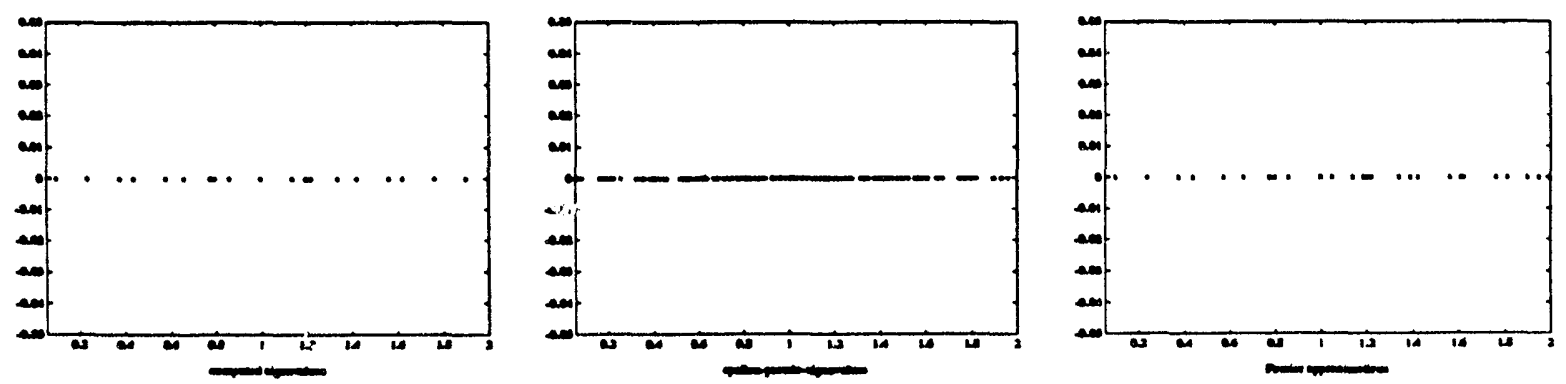

Jacobi preconditioned system
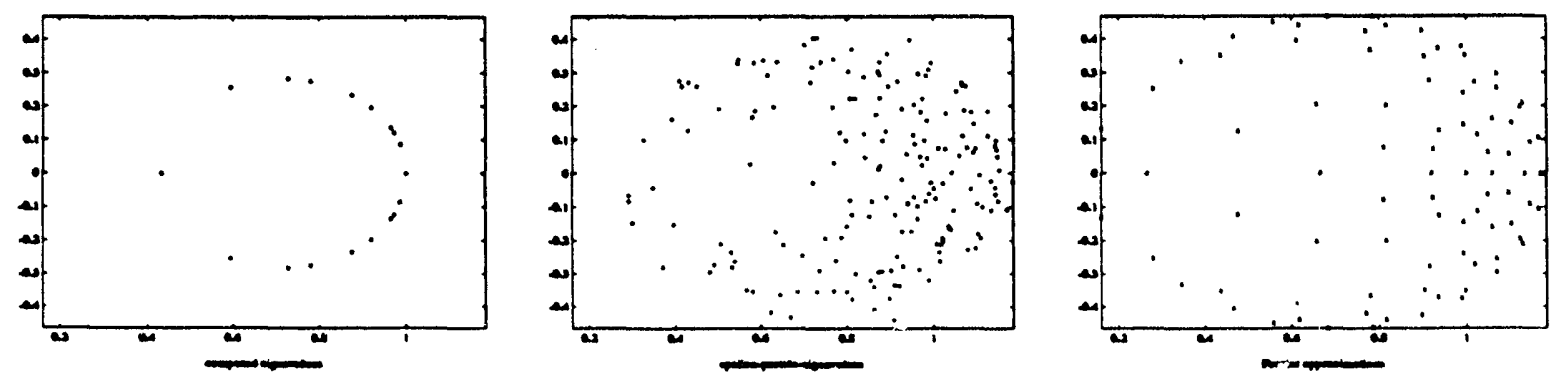

Preconditioned system using SOR splitting matrix
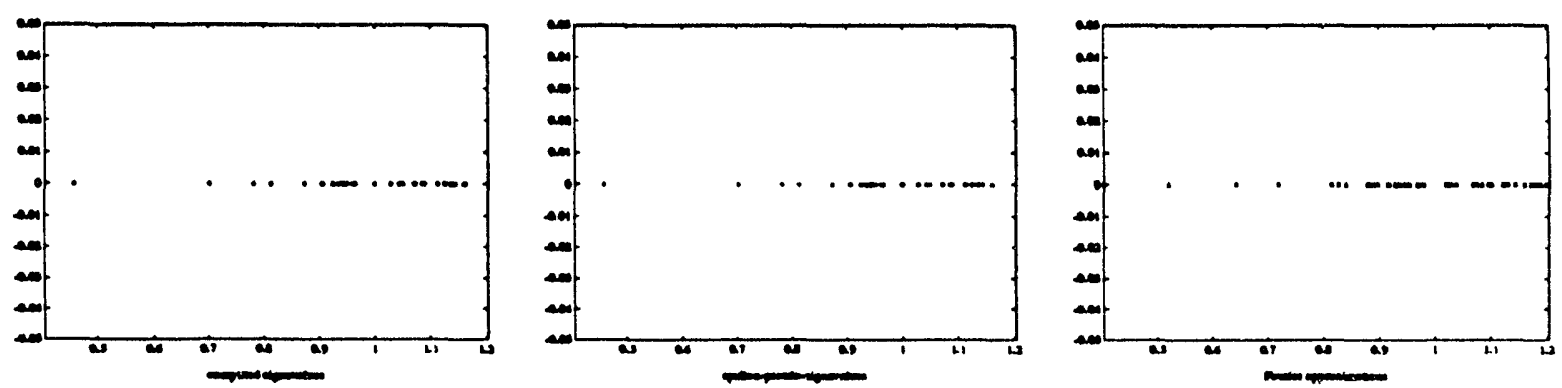

ILU preconditioned system

Figure 7: The computed eigenvalues, $\epsilon$-pseudo-eigenvalues, and Fourier approximate eigenvalues for $M^{-1} A$ using parameter values $\alpha=\beta=0$. 

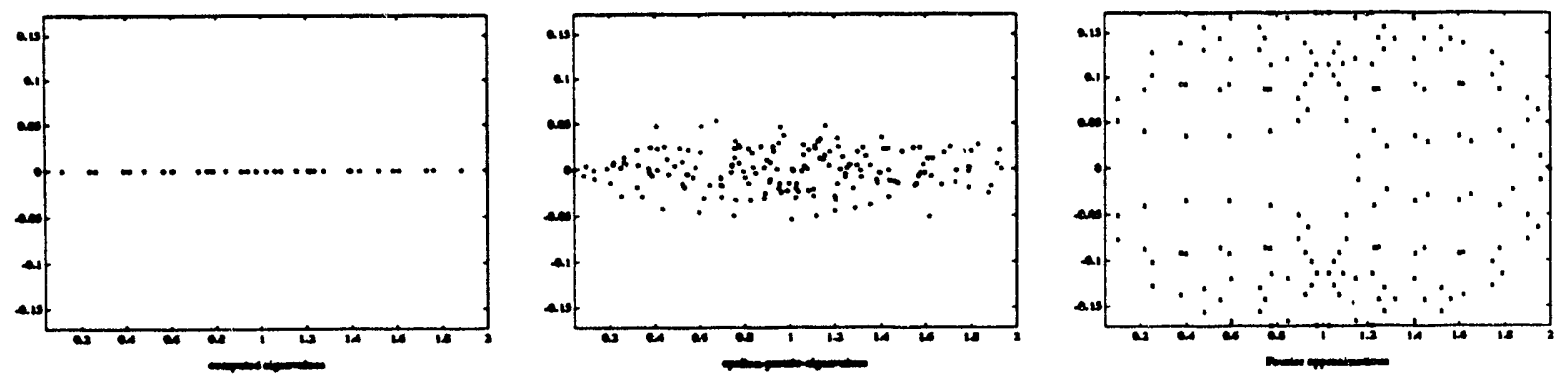

Jacobi preconditioned system
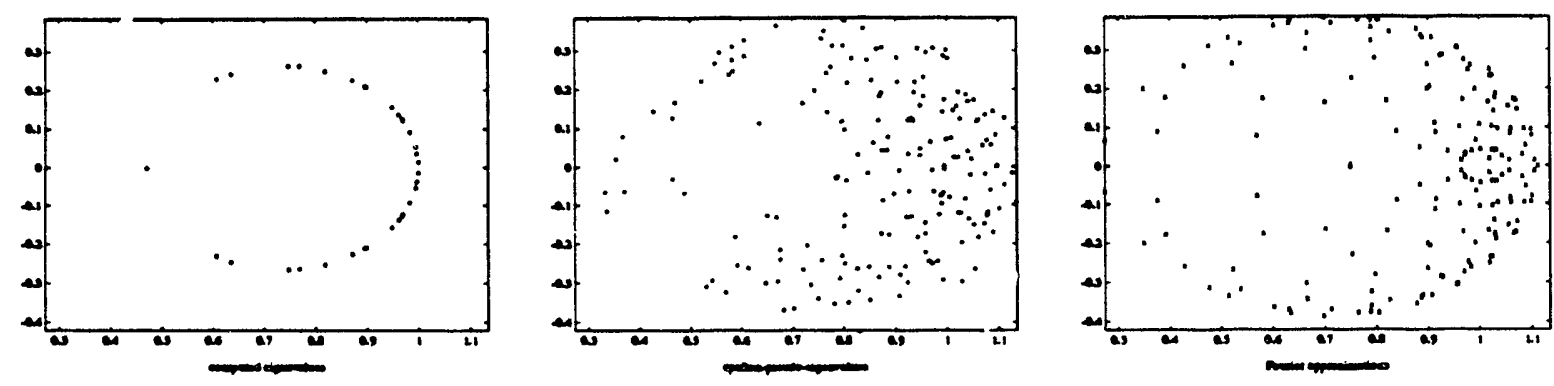

Preconditioned system using SOR splitting matrix
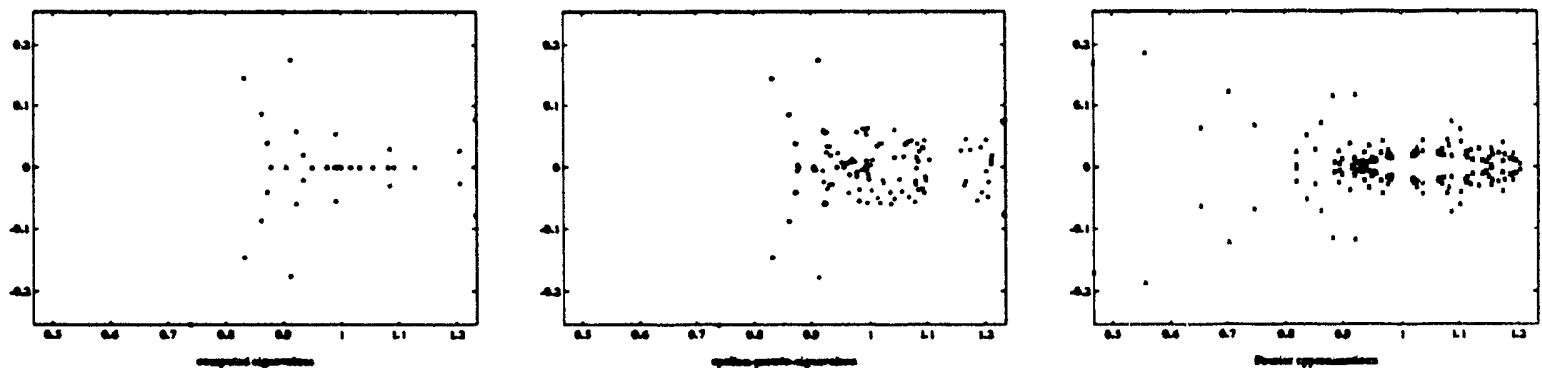

ILU preconditioned system

Figure 8: The computed eigenvalues, $\epsilon$-pseudo-eigenvalues, and Fourier approximate eigenvalues for $M^{-1} A$ using parameter values $\alpha=5, \beta=1$. 

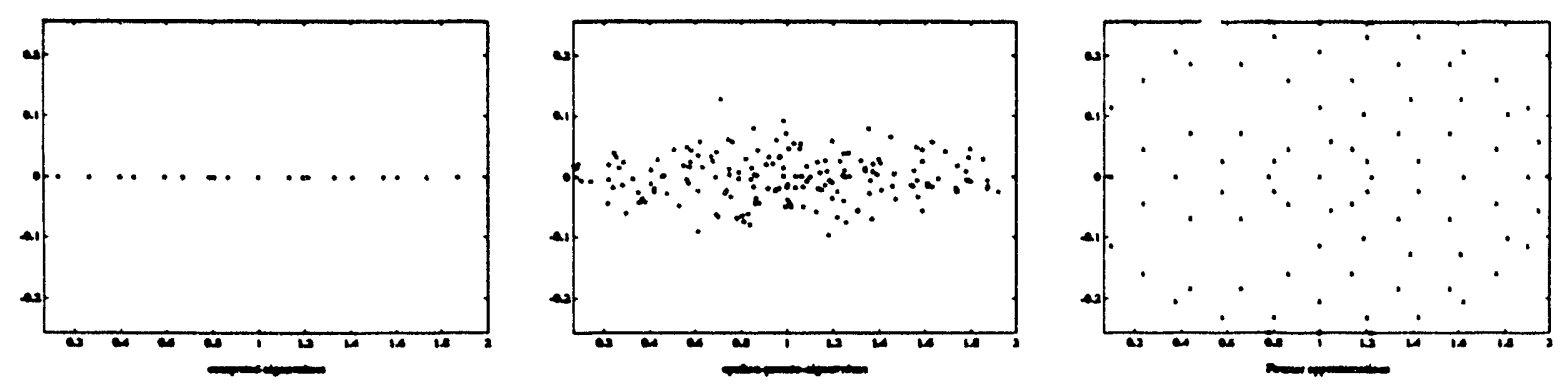

Jacobi preconditioned system
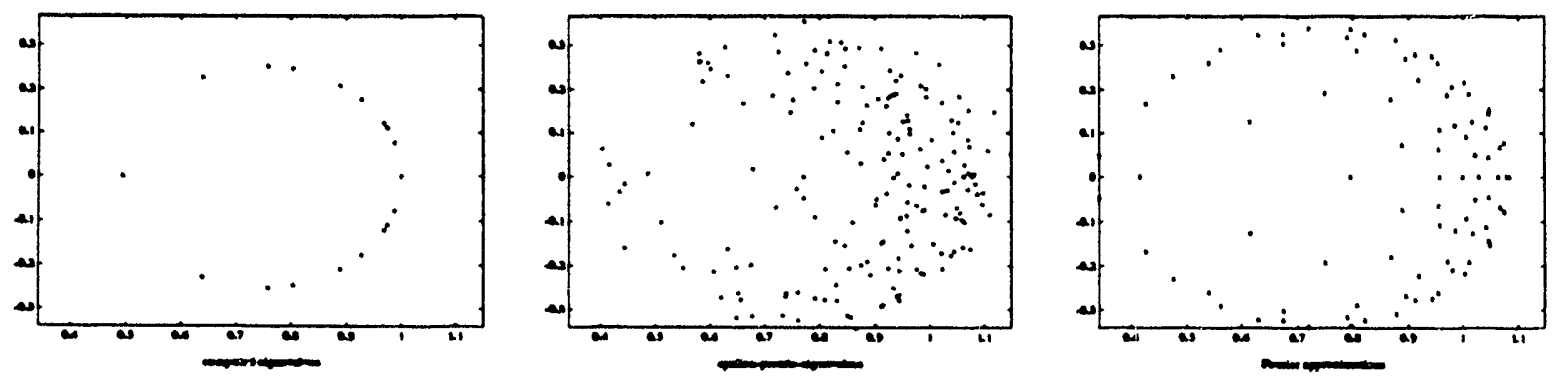

Preconditioned system using SOR splitting matrix
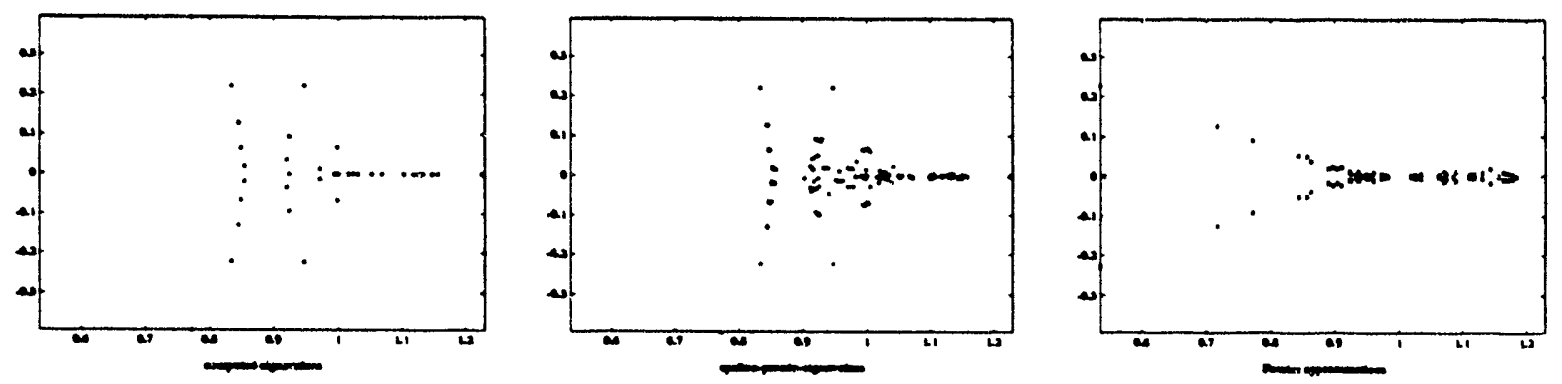

ILU preconditioned system

Figure 9: The computed eigenvalues, $\epsilon$-pseudo-eigenvalues, and Fourier approximate eigenvalues for $M^{-1} A$ using parameter values $\alpha=5, \beta=5$. 


\section{Summary}

The Fourier technique used in the analysis of iterative methods and preconditioners is only an heuristic for nonnormal matrices, yet it yields estimates of extremal eigenvalues and condition numbers that are useful in predicting the behavior of iterative methods and preconditioners.

For Toeplitz matrices a connection between the Fourier analysis technique and $\epsilon$-pseudo-eigenvalues regions has been demonstrated. The boundary of the Fourier approximate eigenvalues is the limiting case of the $\Omega_{r}$ and $\Omega^{R}$ boundary regions for $\epsilon$-pseudo-eigenvalues.

The theory of $\epsilon$-pseudo-eigenvalues of Trefethen not only yields reasons why $\epsilon$ pseudo-eigenvalues are more crucial than eigenvalues for analysis methods for nonhermitian matrices, it also lends credence to the usefulness of the Fourier analysis technique.

For non-Toeplitz matrices the connection between the $\epsilon$-pseudo-eigenvalues and the Fourier approximate eigenvalues is not clear. For the preconditioned systems examined herein, we see that the Fourier approximate eigenvalues do not form an envelop around the $\epsilon$-pseudo-eigenvalues. They do, however, capture some of the clustering behavior of the the $\epsilon$-pseudo-eigenvalues along with estimates of extremal bounds.

\section{References}

[1] L.M. Adams, R.J. LeVeque, and D.M. Young, Analysis of the SOR Iteration for the 9-Point Laplacian, SIAM J. Sci. Stat. Comp., Vol. 25 (5), October 1988.

[2] O. Axelsson, Bounds of Eigenvalues of Preconditioned Matrices, SIAM J. Sci. Matrix Anlaysis, Vol. 13 (3), July 1992.

[3] R.E. Bank, T.F. Chan, W.M. Coughran, Jr. and R.K. Smith, The Alternate-BlockFactorization Procedure For Systems of Partial Differential Equations, BIT, Vol. 29, 1989, pp. 938-954.

[4] A. Brandt, Rigorous Local Mode Analysis of Multigrid, Weizmann Inst. of Sci, Dept. of Applied Math. \& Comp. Sci., Rehovot, Israel, Dec. 1988.

[5] T.F. Chan, Fourier Analysis of Relaxed Incomplete Factorization Preconditioners, SIAM J. Sci. Stat. Comp. 12 (1991), No. 3, May 1991, pp. 668-680.

[6] T.F. Chan and H.C. Elman, Fourier Analysis of Iterative Methods for Elliptic Problems, SIAM Review, Vol. 31, No. 1, March 1989, pp. 20-49.

[7] T.F. Chan and G. Meurant, Fourier Analysis of Block Preconditioners, UCLA CAM Report 90-04, Feb. 1990.

[8] J.M. Donato, and T.F. Chan, Fourier A nalysis of Incomplete Factorization Preconditioners for Three-Dimensional Anisotropic Problems, SIAM J. Sci. Stat. Comp., Vol. 13, No. 1, Jan. 1992. 
[9] T. Dupont, R.P. Kendall, and H.H. Rachford, An Approximate Factorization Procedure For Solving Self-Adjoint Elliptic Difference Equations, SIAM J. of Num. Anal., Vol. 5, No. 3, Sept. 1968.

[10] H.C. Elman, A Stability Analysis of Incomplete LU Factorizations, J. Math. Comp., Vol. 47, No. 175, July 1986, pp. 191-217.

[11] G.H. Golub and C.F. Van Loan, Matrix Computations, Johns Hopkins University Press, 1989.

[12] L.A. Hageman and D.M. Young, Applied Iterative Methods, Academic Press, New York, 1981.

[13] R.J. LeVeque and L.N. Trefethen, Fourier Analysis of the SOR Iteration, IMA J. Numer. Anal. 8 (1988), 273-279.

[14] N.M. Nachtigal, S.C. Reddy, and L.N. Trefethen, How Fast are Nonsymmetric Matrix Iterations? SIAM J. Matrix Anal., Vol. 13, No. 3, July 1992, pp. 778-795.

[15] L. Reichel and L.N. Trefethen, Eigenvalues and Pseudo-Eigenvalues of Toeplitz Matrices, Lin. Alg. Applics., Vol. 162-164, 1992, pp. 153-185.

[16] R.D. Richtmyer and K.W. Morton, Difference Methods for Initial-Value Problems, John Wiley \& Sons, Inc., 1967.

[17] L.N. Trefethen, Algorithms and Analysis for Non-Normal Matrices and Operators, April 30, 1991.

[18] L.N. Trefethen, Approximation Theory and Numerical Linear Algebra, Dec. 1988, Algorithms for Approximation II, J.C. Mason and M.G. Cox (Editors), Chapman, London, to appear.

[19] D.M. Young, Iterative Solution of Large Linear Systems, Academic Press, 1971. 


\section{Appendix}

In this appendix we derive the Fourier approximate eigenvalue expressions for the matrices used in the section on non-Toeplitz examples. Fortunately, the stencil for each of these matrices is a form of seven-point stencil. This allows us to apply the Fourier technique to each of the preconditioned systems using a general form.

We denote the seven-point stencil operator by

$$
M_{7}=\left[\begin{array}{ccc}
f & c & \cdot \\
d & a & b \\
\cdot & \ell & g
\end{array}\right]
$$

The Fourier approximate eigenvalues for this seven-point operator are given by

$$
\hat{\lambda}_{s, t}\left(M_{7}\right)=a+b e^{i \theta_{s}}+c e^{i \phi_{t}}+d e^{-i \theta_{s}}+\ell e^{-i \phi_{t}}+f e^{-i\left(\theta_{s}-\phi_{t}\right)}+g e^{i\left(\theta_{s}-\phi_{t}\right)} .
$$

The Fourier approximate eigenvalues for the matrix $M^{-1} A$ is computed via

$$
\hat{\lambda}_{s, t}\left(M^{-1} A\right)=\frac{\hat{\lambda}_{s, t}(A)}{\hat{\lambda}_{s, t}(M)} .
$$

This follows because the underlying premise of the Fourier technique is that we have circulant matrices, which would both have the Fourier vectors as their eigenvectors.

Let us write the matrix $A$ in terms of its stencil form:

$$
A=\left[\begin{array}{ccc}
\cdot & c & \cdot \\
d & a & b \\
\cdot & \ell & \cdot
\end{array}\right]=\left[\begin{array}{ccc}
\cdot & -1 & \cdot \\
-1-\alpha h & 4+(\alpha+\beta) h & -1 \\
\cdot & -1-\beta h & \cdot
\end{array}\right]
$$

The Fourier approximate eigenvalues for $A$ are then given by:

$$
\begin{aligned}
\hat{\lambda}_{s, t}(A) & =4+(\alpha+\beta) h-e^{i \theta_{s}}-e^{i \phi_{t}}-(1+\alpha h) e^{-i \theta_{s}}-(1+\beta h) e^{-i \phi_{t}} \\
& =4\left(\sin ^{2} \frac{\theta_{s}}{2}+\sin ^{2} \frac{\phi_{t}}{2}\right)+(\alpha+\beta) h-\alpha h e^{-i \theta_{s}}-\beta h e^{-i \phi_{l}}
\end{aligned}
$$

Let us write the matrix $A$ as $A=D+L+U$ where $D$ is a diagonal matrix, $L$ is the strictly lower triangular part of $A$, and $U$ is the strictly upper triangular part of $A$. The Jacobi and SOR matrices can be easily written in terms of these matrices submatrices of $A$. For background on splittings and preconditioners see [11].

Jacobi splitting matrix or preconditioner:

$$
\begin{aligned}
& M=D=\left[\begin{array}{ccc}
\cdot & \cdot & \cdot \\
\cdot & 4+(\alpha+\beta) h & \cdot \\
\cdot & \cdot & \cdot
\end{array}\right] \\
& \hat{\lambda}_{s, t}(M)=4+(\alpha+\beta) h .
\end{aligned}
$$


$\operatorname{SOR}(w)$ splitting matrix:

$$
\begin{aligned}
& M=D+w L=\left[\begin{array}{ccc}
\cdot & \cdot & \cdot \\
-w(1+\alpha h) & 4+(\alpha+\beta) & \cdot \\
\cdot & -w(1+\beta h) & \cdot
\end{array}\right] \\
& \hat{\lambda}_{s, t}(M)=4+(\alpha+\beta) h-w(1+\alpha h) e^{-i \theta_{s}}-w(1+\beta h) e^{-i \phi_{t}} .
\end{aligned}
$$

For the incomplete factorization preconditioner (ILU), the goal is to approximately factor the matrix into the product of a lower $(L)$ and upper $(U)$ triangular matrices where the triangular matrices have the same sparsity pattern as the original matrix. Let $M=L U$ represent the ILU preconditioner. We also require that the corresponding entries of $M$ and $A$ are equal whenever the entry in $A$ is non-zero.

The ILU preconditioner for the five-point stencil is then given by

$$
\begin{aligned}
M=L U & =\left[\begin{array}{ccc}
\cdot & \cdot & \\
d & \alpha_{j k} & \cdot \\
\cdot & \ell & \cdot
\end{array}\right]\left[\begin{array}{ccc}
\cdot & \alpha_{j k}^{-1} c & \cdot \\
\cdot & 1 & \alpha_{j k}^{-1} b \\
& \cdot & \cdot
\end{array}\right] \\
& =\left[\begin{array}{ccc}
m_{j-1, k+1} & c & \cdot \\
d & m_{j, k} & b \\
\cdot & \ell & m_{j+1, k-1}
\end{array}\right] .
\end{aligned}
$$

The entries $m_{j-1, k+1}=c d / \alpha_{j-1, k}$ and $m_{j+1, k-1}=b \ell / \alpha_{j, k-1}$ are called fillins because they occur in locations corresponding to places where the original matrix $A$ had zero entries. For the center element of $M$ we have the recurrence

$$
m_{j, k}=\alpha_{j k}+b d / \alpha_{j-1, k}+c \ell / \alpha_{j, k-1} .
$$

Following the steps in [6], we use an asymptotic value $\alpha$ for the $\alpha_{j, k}$ values. This is necessary since we need the entries along a given diagonal to be a constant value to use the Fourier technique.

For ILU, $m_{j k}=a$ and the asymptotic value for the $\alpha_{j, k}$ is $\alpha=a-b d-c \ell$. The asymptotic values for the fillins are then given by $m_{j-1, k+1}=c d / \alpha$ and $m_{j+1, k-1}=$ $b \ell / \alpha$. Hence, for analysis purposes, we use the asymptotic version of the matrix $M$ :

$$
\begin{aligned}
& M=\left[\begin{array}{ccc}
c d / \alpha & c & \cdot \\
d & a & b \\
\cdot & \ell & b \ell / \alpha
\end{array}\right]=\left[\begin{array}{ccc}
\cdot & c & \cdot \\
d & a & b \\
\cdot & \ell & \cdot
\end{array}\right]+\left[\begin{array}{ccc}
c d / \alpha & \cdot & \cdot \\
\cdot & \cdot & \cdot \\
\cdot & \cdot & b \ell / \alpha
\end{array}\right] \\
& \hat{\lambda}_{s, t}(M)=\hat{\lambda}_{s, t}(A)+\frac{1}{\alpha}\left(c d e^{-i\left(\theta_{s}-\phi_{t}\right)}+b \ell e^{i\left(\theta_{s}-\phi_{t}\right)}\right) .
\end{aligned}
$$




\section{INTERNAL DISTRIBUTION}

1. B. R. Appleton

2-3. T. S. Darland

4. E. F. D'Azevedo

5-9. J. M. Donato

10. J. J. Dongarra

11. G. A. Geist

12. L. J. Gray

13. M. R. Leuze

14. E. G. $\mathrm{Ng}$

15. C. E. Oliver

16. B. W. Peyton

17-21. S. A. Raby
22. C. H. Romine

23. T. H. Rowan

24. B. D. Semeraro

25-29. R. F. Sincovec

30-34. R. C. Ward

35. P. H. Worley

36. Central Research Library

37. ORNL Patent Office

38. K-25 Appl Tech Library

39. Y-12 Technical Library

40. Lab Records Dept - RC

41-42. Laboratory Records Dept

\section{EXTERNAL DISTRIBUTION}

43. Steven Ashby, Lawrence Livermore National Laboratory, P.O. Box 808, L-316, Livermore, CA 94551

44. Jesse L. Barlow, Department of Computer Science, Pennsylvania State University, University Park, PA 16802

45. Michael Berry, Department of Computer Science, Ayres Hall, University of Tennessee, Knoxville, TN 37996-1301

46. Jean R. S. Blair, Department of Computer Science, Ayres Hall, University of Tennessee, Knoxville, TN 37996-1301

47. Roger W. Brockett, Pierce Hall 29 Oxford Street Harvard University Cambridge, MA 02138

48. Daniela Calvetti, Stevens Inst of Technology, Department of Mathematics, Hoboken NJ 07030

49. Tony Chan, Department of Mathematics, University of California, Los Angeles, 405 Hilgard Avenue, Los Angeles, CA 90024

50. Alexandre Chorin, Mathematics Department, Lawrence Berkeley Laboratory, Berkeley, CA 94720

51. James Corones, Ames Laboratory, lowa State University, Ames, IA 50011

52. Rosa Donat, Dept. Matematica Aplicada i Astronomia, Facultat de Matematiques, C/ Dr. Moliner, 50, 46100 Burjassot (Valencia) Spain

53. Donald J. Dudziak, Department of Nuclear Engineering, 110B Burlington Engineering Labs, North Carolina State University, Raleigh, NC 27695-7909

54. Howard C. Elman, Computer Science Department, University of Maryland, College Park, MD 20742

55. Richard E. Ewing, Department of Mathematics, Texas A\&M University, College Station, TX 77843 
56. James Glimm, Department of Mathematics, State University of New York, Stony Brook, NY 11794

57. Gene H. Golub, Department of Computer Science, Stanford University, Stanford, CA 94305

58. Per Christian Hansen, UCI*C Lyngby, Building 305, Technical University of Denmark, DK-2800 Lyngby, Denmark

59. Michael T. Heath, National Center for Supercomputing Applications, 4157 Beckman Institute, University of Illinois, 405 North Mathews Avenue, Urbana, IL $61801-2300$

60. Fred Howes, Office of Scientific Computing, ER-7, Applied Mathematical Sciences, Office of Energy Research, Department of Energy, Washington, DC 20585

61. Gary Johnson, Office of Scientific Computing, ER-7, Applied Mathematical Sciences, Office of Energy Research, Department of Energy, Washington, DC 20585

62. Malvyn H. Kalos, Cornell Theory Center, Engineering and Theory Center Bldg., Cornell University, Ithaca, NY 14853-3901

63. Hans Kaper, Mathematics and Computer Science Division, Argonne National Laboratory, 9700 South Cass Avenue, Bldg. 221, Argonne, IL 60439

64. Linda Kaufman, Bell Laboratories, 600 Mountain Avenue, Murray Hill, NJ 07974

65. David Keyes, Mechanical Engineering Dept., Yale University, P.O. Box 2159, Yale Station, New Haven, CT 06520-2159

66. David Kincaid, Center for Numerical Analysis, RLM 13-150, Austin, TX 78712

67. Peter D. Lax, Courant Institute of Mathematical Sciences, New York University, 251 Mercer Street, New York, NY 10012

68. James E. Leiss, Rt. 2, Box 142C, Broadway, VA 22815

69. Juan Meza, Sandia National Laboratories, Division 8211, P.O. Box 969, Livermore, CA 94551-0969

70. Neville Moray, Department of Mechanical and Industrial Engineering, University of Illinois, 1206 West Green Street, Urbana, IL 61801

71. David Nelson, Director of Office of Scientific Computing Staff, ER-7, Applied Mathematical Sciences, Office of Energy Research, Department of Energy, Washington, DC 20585

72. Dianne P. O'Leary, Computer Science Department, University of Maryland, College Park, MD 20742

73. Julia Olkin, Remote Measurement Lab., SRI International \#301-66, 333 Ravenswood Ave., Menlo Park, CA 94025-3493

74. Beth Ong, University of California, San Diego, Department of Mathematics, 9500 Gilman Drive, La Jolla, CA 92093-0112

75. James M. Ortega, Department of Applied Mathematics, Thornton Hall, University of Virginia, Charlottesville, VA 22901

76. Jesse Poore, Department of Computer Science, Ayres Hall, University of Tennessee, Knoxville, TN 37996-1301 
77. Lothar Reichel, Kent State University, Dept. of Mathematics and Computer Science, Kent, $\mathrm{OH} 44242$

78. Horst Simon, Mail Stop T045-1 NASA Ames Research Senter, Moffett Field, CA 94035

79. G. W. Stewart, Computer Science Department, University of Maryland, College Park, MD 20742

80. Charles Tong, Dept. 8210, Sandia National Labs., Livermore, CA 94551-0969

81. Lloyd Nicholas Trefethen, Cornell University, Dept. of Computer Science, Ithaca, NY 14853

82. Mary F. Wheeler, Rice University, Department of Mathematics, P.O. Box 1892, Houston, TX 77251

83. David Young, University of Texas, Center for Numerical Analysis, RLM 13.150, Austin, TX 78731

84. Office of Assistant Manager for Energy Research and Development, Department of Energy, Oak Ridge Operations Office, P.O. Box 2001 Oak Ridge, TN 37831-8600

85-86. Office of Scientific \& Technical Information, P.O. Box 62, Oak Ridge, TN 37831 

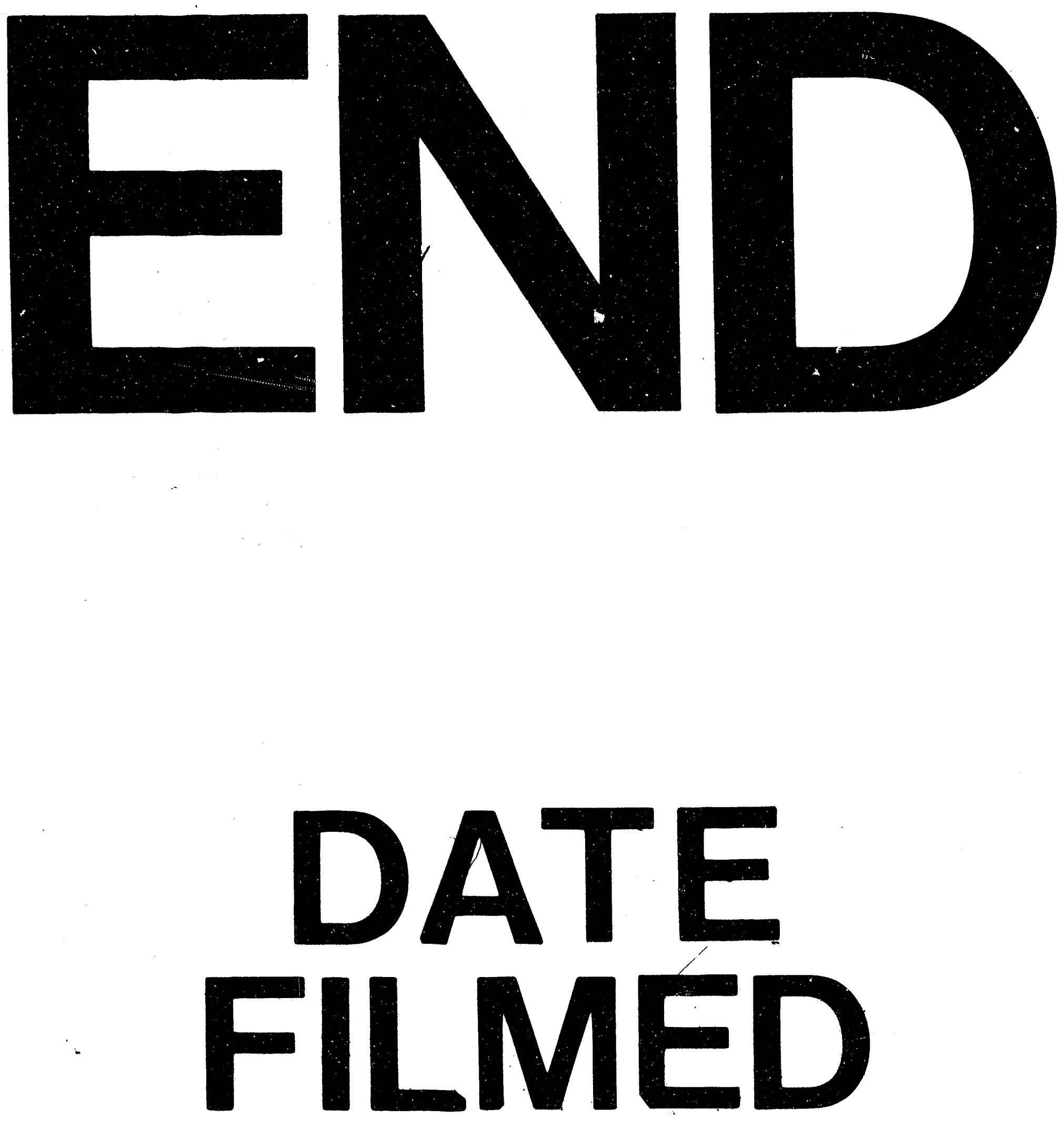

1

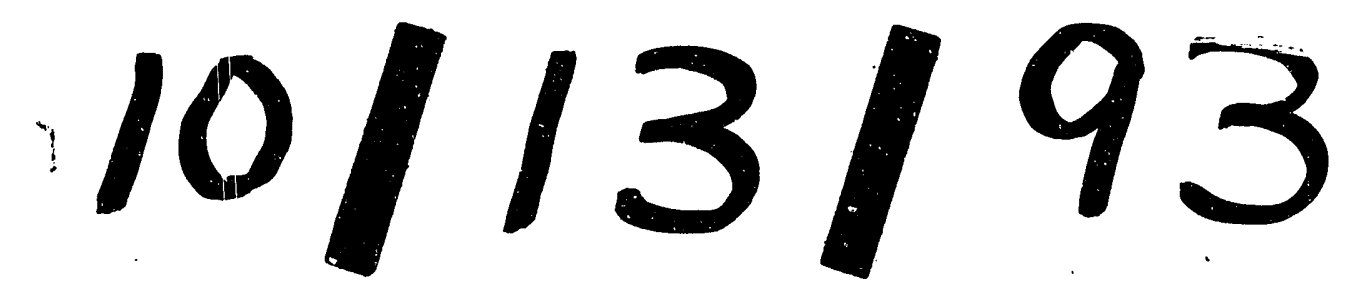

